Relations industrielles

Industrial Relations

\title{
Les préventeurs, par Pierre Caloni, Société d'éditions françaises et internationales, Paris, 1960, 437 pp.
}

\section{Gaston Cholette}

Volume 16, numéro 2, avril 1961

URI : https://id.erudit.org/iderudit/1021818ar

DOI : https://doi.org/10.7202/1021818ar

Aller au sommaire du numéro

Éditeur(s)

Département des relations industrielles de l’Université Laval

ISSN

0034-379X (imprimé)

1703-8138 (numérique)

Découvrir la revue

Citer ce compte rendu

Cholette, G. (1961). Compte rendu de [Les préventeurs, par Pierre Caloni, Société d'éditions françaises et internationales, Paris, 1960, 437 pp.] Relations industrielles / Industrial Relations, 16(2), 273-273.

https://doi.org/10.7202/1021818ar

Tous droits réservés @ C Département des relations industrielles de l’Université Laval, 1961
Ce document est protégé par la loi sur le droit d'auteur. L'utilisation des services d'Érudit (y compris la reproduction) est assujettie à sa politique d'utilisation que vous pouvez consulter en ligne.

https://apropos.erudit.org/fr/usagers/politique-dutilisation/ 
titution. On trouve à la fin de la plupart des chapitres une synthèse comparative.

Le volume se termine par une magnifique conclusion sur la notion de personnalité juridique, reconnue à des titres divers et avec des conséquences plus ou moins grandes dans tous les Etats. L'auteur laisse de côté les théories et constructions purement doctrinales et se réfère seulement aux solutions découlant du droit positif des différents pays. On y trouve notamment un exposé de la conception anglaise et de la conception germanique, autour desquelles gravitent tous les systèmes.

\section{Gaston Cholette.}

The World of Work, Industrial Society and Human Relations, par Robert Dubin, Prentice Hall, Englewood Cliffs N.J., 1958, 448 pp., $\$ 8.00$.

Ce volume porte sur le monde du travail dans la société industrielle américaine. L'étude a pour objet les comportements des divers agents qui évoluent dans ce monde complexe et leurs relations. La question des relations patronales-syndicales est délibérément laissée de côté, car l'auteur a oru bon, à cause de son importance, de lui consacrer un autre ouvrage spéciel (Working Union-Management Relations, Englewood Cliffs, Prentice Hall, 1958). Ces deux ouvrages se complètent et peuvent etre lues séparément ou être considérés comme les deux tomes d'un même traité.

L'auteur projette sur les problèmes qu'il considère un éclairage original au moyen de deux disciplines appropriées: la sociologie et la psychologie. Il observe et analyse une quantité énorme de faits scientifiquement contrôlés. Il cherche à découvrir ce que les gens font lorsqu'ils travaillent et les raisons, les mobiles qui sont à l'origine de leurs actes.

Le volume est divisé en cinq parties principales: une introduction sur le travail dans la société moderne, une deuxième partie sur les structures du monde du travail, une troisième sur la population ouvrière, une quatrième sur Porganisation, la mise en oeuvre des forces du travail, enfin une cinquième partie sur la direction des institutions da monde du travail.

Ceux qui s'intéressent aux relations du travail auront profit à parcourir ce volume et à l'utiliser pour mieux comprendre et résoudre un grand nombre de problèmes qui se posent dans leur activité professionnelle.

\section{Gaston Chotette.}

Les préventeurs, par Pierre Caloni, Société d'éditions françaises et internationales, Paris, 1960, 437 pp.

Ce livre porte sur la lutte contre les accidents du travail. Son titre un peu surprenant désigne la personne dont l'occupation principale est la prévention.

Il s'agit de l'histoire d'une réalisation personnelle de l'auteur dans les professions du bâtiment et des travaux publics. L'auteur raconte la tâche qu'il a entreprise à son retour de la guerre de 1914-1918 et qu'il a poursuivie jusqu'à aujourd'hui. C'est donc par le biais d'une expérience personnelle de l'auteur que le lecteur pénètre dans le monde de la prévention des accidents du travail dans le bâtiment et les travaux publics, à travers le dédale des obstacles administratifs et des changements de politique.

Ce qui intéressera tout spécialement ceux qui s'occupent de la prévention des accidents du travail dans la province de Québec et au Canada en général, c'est la description de la structure et des rouages des institutions qui ont été édifiées en France, où une collaboration organique entre ouvriers, syndicats, employeurs et fonctionnaires publics donne d'excellents résultats.

L'auteur consacre une partie de son ouvrage à des réflexions sur la nature humaine et ses réactions devant le risque, et sur la meilleure manière de comprendre la tâche de préventeur.

On trouve enfin, dans la dernière partie, une imposante série dannexes qui fournissent une abondante documentation. 